ARTICLE

Received 6 Oct 2014 | Accepted 28 Jul 2015 | Published 15 Sep 2015

DOI: $10.1057 /$ palcomms.2015.22

OPEN

\title{
Competitive dynamics between criminals and law enforcement explains the super-linear scaling of crime in cities
}

Soumya Banerjee ${ }^{1,2,3,4,5}$, Pascal Van Hentenryck ${ }^{6}$ and Manuel Cebrian ${ }^{6}$

\begin{abstract}
While cities have been the engine for innovation and growth for many millennia, they have also endured disproportionately more crime than smaller cities. Similarly to other urban sociological quantities, such as income, gross domestic product (GDP) and number of granted patents, it has been observed that crime scales super-linearly with city size. The default assumption is that super-linear scaling of crime, like other urban attributes, derives from agglomerative effects (that is, increasing returns from potentially more productive connections among criminals). However, crime initiation appears to be generated linearly with the population of a city, and the number of law enforcement officials scales sublinearly with city population. We hypothesize that the observed scaling exponent for net crime in a city is the result of competing dynamics between criminals and law enforcement, each with different scaling exponents, and where criminals win in the numbers game. We propose a simple dynamical model able to accommodate these empirical observations, as well as the potential multiple scaling regimes emerging from the competitive dynamics between crime and law enforcement. Our model is also general enough to be able to correctly account for crime in universities, where university crime does not scale super-linearly, but linearly with enrolment size.
\end{abstract}

\footnotetext{
${ }^{1}$ Department of Biostatistics, Harvard School of Public Health, Boston, USA ${ }^{2}$ The Broad Institute of MIT and Harvard, Cambridge, USA ${ }^{3}$ Complex Biological Systems Alliance, North Andover, USA ${ }^{4}$ Ronin Institute, Montclair, USA 5 Harvard Medical School, Boston, USA ${ }^{6}$ National Information and Communications Technology Australia, Melbourne, Australia Correspondence: (email: neel.soumya@gmail.com)
} 


\section{Introduction}

ities triumph as super-linear hubs of innovation, productivity and energy efficiency (Bettencourt et al., 2007; Batty, 2008; Bettencourt and West, 2010; Glaeser, 2011). However, as cities grow in size, crime rates also increase superlinearly (Glaeser and Sacerdote, 1996; Cullen and Levitt, 1999; Gould et al., 2002), confirming the stereotype that per capita rates of crime are much higher in a large, hectic city like New York than in smaller, more peaceful Santa Fe.

Urban data obtained at multiple levels of resolutions, ranging from infrastructure to social networks, has prompted mathematical models that explain the positive agglomerative effects of cities: larger cities essentially have human networks that are super-linearly more connected, and information and ideas flow faster and wider across the population, boosting innovation (Schläpfer et al., 2014; Bettencourt, 2013; Pan et al., 2013). However, the super-linear scaling relationship observed for crime has not yet been given a satisfactory generative mechanism. Two main hurdles may be preventing theorists from coming up with successful explanations.

First, it is unclear that criminals would be able to exploit these enhanced inter-personal urban networks for increased collaborations in larger cities. Crime is a strongly local phenomenon (Keizer et al., 2008; Short et al., 2010; Salesses et al., 2013); the spreading of crime and disorder has strong geographical decay, limiting the benefits of a large city (Keizer et al., 2008); and most criminals are not creative professionals, and hence their capacity to mature and specialize in information-rich environments is bounded (Tumminello et al., 2013). Second, if a theory can be developed to explain the scaling of crime, it must incorporate the competitive dynamics between criminals and law enforcement. Crime initiation/perpetration and police intervention while crime is in progress, happen on approximately the same timescale and therefore crime can be in some cases stopped before being perpetrated. Criminals are indeed involved in an arms race with law enforcement (Baudains et al., 2013a, b; Davies et al., 2013). A higher patrolling efficiency or higher numbers of police may reduce the number of crimes, not necessarily by reducing the number of individuals likely to commit a crime, but by ultimately preventing crime from being successfully completed.

In this work, we report that the main ingredients affecting net crime, namely crime and law enforcement, display notably different scaling relationships with human agglomeration size. On the one hand, initiation of crime scales linearly with city size, whereas the size of the police force scales sublinearly. We observe that crime in cities exhibits a super-linear scaling with city population. We hypothesize that this is due to the fact that criminals and police display different scaling relationships.

Crime has been modelled using mathematical models like ordinary differential equation models (McMillon et al., 2014), partial differential equation models (Baudains et al., 2013c; D'Orsogna and Perc, 2014) and spatial and agent-based models (Short et al., 2010; Baudains et al., 2013c; Davies et al., 2013; Perc et al., 2013). Our approach is to use differential equation models that capture the competitive dynamics between law enforcement and criminals and apply them to large-scale data on crime in cities worldwide. For more we refer readers to the recent reviews on the modelling of crime using different mathematical techniques (D'Orsogna and Perc 2014; Helbing et al., 2014).

We model crime mathematically as an arms race between criminals and police. Our model predicts that the super-linear scaling of crime in cities is a direct consequence of the mathematical model and the fact that the number of police scales sublinearly with city size. On the other hand, we observe that crimes on university campuses exhibit a different, sublinear relationship with university size. Net crime initiation is sublinear in campus enrolment size, with also a sublinear scaling of oncampus police. This prompts us to consider whether super-linear scaling of crime is in fact a universal feature of human agglomeration, or just one of the multiple scaling regimes emerging from the interaction between criminals and law enforcement. A successful theory for the scaling of crime in cities should be able to separately model both the endogenous rates of crime generation, as well as the size and efficacy of law enforcement, tying them together in a coherent mathematical model able to reproduce empirical data. A more mechanistic understanding of how crime scales with human agglomerations may help inform public policy, law enforcement resource allocation, and planning decisions for urban settings and other large-scale human agglomerations.

\section{Methods}

Data sources. The datasets that were analysed have been made publicly available in the Dataverse repository (Banerjee et al., 2015). These datasets were derived from detailed data on crime occurrences from publicly available datasets both in the UCI Machine Learning Repository (United States Department of Commerce, Bureau of the Census, Census of Population and Housing 1990 United States: Summary Tape File 1a \& 3a (Computer Files), 1990; United States Department of Justice, Bureau of Justice Statistics, Law Enforcement Management and Administrative Statistics (Computer File), 1992; Redmond and Baveja, 2002; Lichman, 2013) and the FBI Uniform Crime Report (United States Department of Justice, Federal Bureau of Investigation, Crime in the United States (Computer File), 1995). The datasets contain the number of crimes committed in the United States, number of police in US cities, city population size, number of requests to police, police budget and so on. We note the caveat that the UCI dataset combines data from two different times: sociological data is from the 1990 Census and crime data is from the year 1995. Statistical analysis was carried out with programs written in Matlab (MATLAB and Statistics Toolbox Release, 2012).

\section{Model}

We propose a non-linear dynamical model to simulate crime in cities. The model simulates the arms race between criminals and law enforcement. Let us assume that out of a population of $N$ individuals in a city of area $A$, there are $P_{C}$ criminals, $P_{\mathrm{NC}}$ noncriminals or ordinary citizens, and $P_{\mathrm{LE}}$ law enforcement officials.

The number of criminals per unit area is $P_{C} / A$, and let us denote this as $D_{C}$ (density of criminals). Similarly, the number of ordinary (non-criminals) people per unit area is $P_{\mathrm{NC}} / A$, and let us denote this as $D_{\mathrm{NC}}$ (density of non-criminals). Finally, the number of law enforcement officials per unit area is $P_{\mathrm{LE}} / A$ and let this be denoted by $D_{\mathrm{LE}}$ (density of law enforcement officers). Let the density of crimes be $C$ and the total number of crimes be $C \times A$.

We assume that the number (and hence the density) of normal people (non-criminals), criminals and law enforcement does not change for the time period we are interested in. This assumption can be expected to hold in general unless a city is undergoing major social upheavals, as for example the recent London riots of 2011 (Davies et al., 2013), Arab Spring revolutions of 2011 through 2013, and the Stockholm riots of 2013. Our equations can be extended to simulate a situation where the number of police increases rapidly over a few days in response to a sudden increase in the number of criminals (see Supplementary Information: Model Extensions). The model is additionally justifiable on the grounds that our model is tested against data that was collected over one particular year, and changes in police numbers and population are subsequently ignored in the discussion that follows. We note the caveat that not all noncriminals may be completely innocent.

Similar to previous models (McMillon et al., 2014), we assume that crimes are generated by the interaction of criminals and noncriminals, on which criminals perform a criminal action, with rate constant $\alpha$. Crimes are prevented by law enforcement at a rate proportional to the density of crimes and law enforcement with 
rate constant $\beta$. The model is shown below, and is diagrammatically represented in Fig. 1:

$\frac{\mathrm{dC}}{\mathrm{dt}}=\alpha \cdot D_{C} \cdot D_{N C}-\beta \cdot C \cdot D_{L E}$

The model produces an evolution of the density of crime over time (Supplementary Fig. 1).

We again focus on the steady-state and peak values of crime.

The density of crimes committed at steady-state and at the peak of crimes are given by the following equation (derived by setting equation (1) to 0 ; the derivative of crimes committed will be zero at steady-state and at the time when crimes peak, reflecting the notion that the action of police and criminals balance each other).

$C_{S S}=\frac{\alpha \cdot D_{C} \cdot D_{N C}}{\beta \cdot D_{L E}}$

Since we are interested in the actual number of crimes in a city, after converting from densities to numbers we get:

Crime $_{S S}=\frac{\alpha}{\beta} \times \frac{N_{\text {criminals }} \cdot N_{\text {non-criminals }}}{N_{\text {police }}}$

where $N_{\text {criminals }}$ is the number of criminals, $N_{\text {non-criminals }}$ is the number of non-criminals, and $N_{\text {police }}$ is the number of law enforcement officials in a city.

Since we are also interested in how the number of crimes scale with the population of a city $(N)$, let us assume that $N_{\text {normal }} \propto N$, $N_{\text {criminals }} \propto N^{x_{\text {crime }}}$, and $N_{\text {police }} \propto N^{y_{\text {police }}}$ (where $x_{\text {crime }}$ and $y_{\text {police }}$ are the scaling exponents for how the number of criminals and the number of police scales with population size, respectively). This gives us the following scaling relation for the number of crimes in

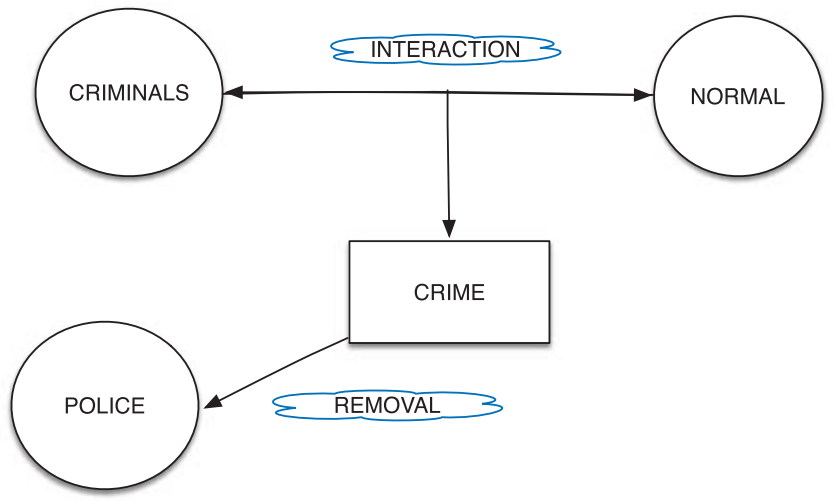

Figure 1 | An interaction diagram showing the arms race between criminals and police.

Crime is the product of the interaction between criminals and normal people (non-criminals). Police try to stop crimes, arresting its further spread. a city of population $N$ :

Crime $_{S S} \propto N^{1+\left(x_{\text {crime }}-y_{\text {police }}\right)}$

The scaling equation (equation (4)) gives us three possibilities for how crime can scale.

1. $x_{\text {crime }}>y_{\text {police }}$ If the scaling exponent for criminals is greater than that of law enforcement, this will lead to super-linear scaling of crime in a city: Crime ${ }_{\text {ss }} \propto N^{\text {exponent }}$ where exponent is greater than 1 .

2. $x_{\text {crime }}=y_{\text {police }}$. If the scaling exponent for criminals is equal that of law enforcement, this will lead to linear scaling of crime in a city: Crime ${ }_{s s} \propto N^{\text {exponent }}$ where exponent is equal to 1 .

3. $x_{\text {crime }}<y_{\text {police }}$. If the scaling exponent for criminals is less than that of law enforcement, this will lead to sublinear scaling of crime in a city: Crime $_{s s} \propto N^{\text {exponent }}$ where exponent is less than 1 .

\section{Results}

Scaling of crime in cities. Our central result, equation (4), predicts that net crime should scale super-linearly whenever $x_{\text {crime }}>y_{\text {police }}$. The scaling relationships and relevant statistics are summarized in Table 1. Empirically we observe that the net number of crimes in a city scales super-linearly with the size of the city (total population of a city) (Fig. 2, ordinary least squares [OLS] slope $=1.26,95 \%$ confidence interval on slope $=[1.22$, $1.30], r^{2}=0.65, P$-value $\left.=1 \mathrm{e}-100\right)$.

Hence crime scales super-linearly with city size. In addition we tested super-linearity using another method used to test allometric scaling (Warton et al., 2006) using the SMATR package in $R$ (Warton et al., 2012). This analysis also confirmed super-linearity (semi-major axis regression slope $=1.56$,

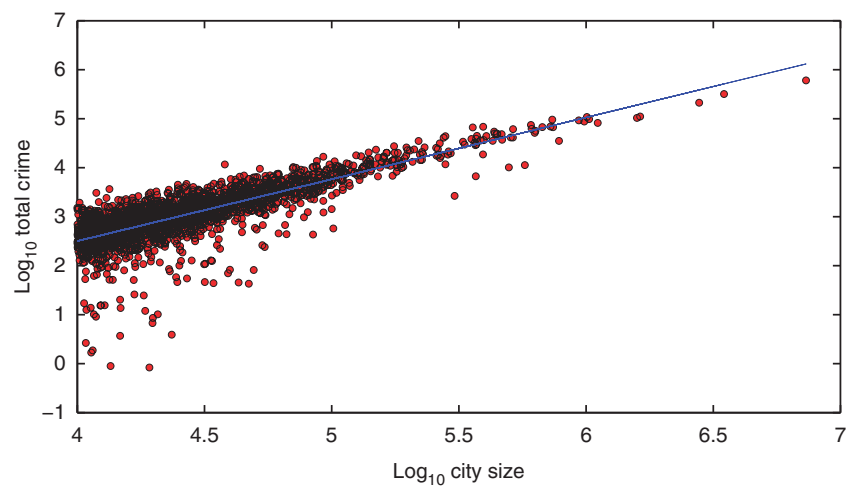

Figure 2 | Super-linear scaling of crime in cities.

Note: Plot showing total number of crimes versus city size (population) from empirical data. OLS slope $=1.26, r^{2}=0.65, P$-value $=1 e-100$.

Table 1 | Scaling relations for crime with relevant statistics

$\log _{10}($ Dependent variable $)=$ Scaling exponent ${ }^{\star} \log _{10}$ (Independent variable)+intercept using ordinary least squares regression

\begin{tabular}{|c|c|c|c|c|}
\hline Dependent variable & Independent variable & Scaling exponent (mean and 95\% Cl) & $r^{2}$ & $P$-value \\
\hline Total crimes in cities & City population & $1.26[1.22,1.30]$ & 0.65 & $1 e-100$ \\
\hline Total requests for police & City population & $0.96[0.88,1.05]$ & 0.60 & $1 e-70$ \\
\hline Sworn police number & City population & $0.82[0.77,0.88]$ & 0.69 & $5 e-88$ \\
\hline Police budget in cities & City population & $0.88[0.81,0.95]$ & 0.65 & $7 e-80$ \\
\hline Total crimes in universities & Enrolment size & $0.82[0.76,0.88]$ & 0.53 & $3 e-98$ \\
\hline Number of police in universities & Enrolment size & $0.56[0.50,0.61]$ & 0.37 & $2 e-66$ \\
\hline
\end{tabular}


$95 \%$ confidence interval on slope $=[1.52,1.60], r^{2}=0.65$, $P$-value $=2 \mathrm{e}-16)$.

However, we observe that crime is generated at numbers which scale linearly with the population of a city (Fig. 3, OLS slope $=0.96$, $95 \%$ confidence interval on slope $=[0.88,1.05], r^{2}=0.60, P$ value $=1 \mathrm{e}-70)$. The number of police officers scales sublinearly with city size (Fig. 4, OLS slope $=0.82,95 \%$ confidence interval on slope $=[0.77,0.88], r^{2}=0.69, P$-value $\left.=5 \mathrm{e}-88\right)$.

Budget for police also increases sublinearly with city population (Fig. 5, OLS slope $=0.88,95 \%$ confidence interval on slope $\left.=[0.81,0.95], r^{2}=0.65\right)$. Hence the relationship of the number of requests for police is linear, whereas the size of the police force is sublinear with city size, resulting in the observed super-linear scaling of net crime.

We also looked at the average overtime worked by police-to check if an increased police effort could make up for smaller police number-and the relationship with city size is barely significant statistically (Supplementary Fig. 2, OLS slope $=0.11$, $r^{2}=0.01, P$-value $\left.=0.04\right)$.

Scaling of crime in universities. We also looked at a different dataset (FBI Uniform Crime Report) that reports number of crimes and police size on US university campuses. The scaling relationships and relevant statistics are again summarized in Table 1 . We observed that net crime scaled sublinearly with size of the university (defined as number of students enroled) (Fig. 6, OLS slope $=0.82,95 \%$ confidence interval on slope $=[0.76,0.88]$, $r^{2}=0.53, P$-value $\left.=3 \mathrm{e}-98\right)$. Also the number of police on campus scaled sublinearly with university size (Fig. 7, OLS slope $=0.56$, $95 \%$ confidence interval on slope $=[0.50,0.61], r^{2}=0.37$, $P$-value $=2 \mathrm{e}-66)$.

Our model predicts that the number of police requests in universities must also have been sublinear (equation (4)), instead of the linear relationship observed in cities-though we have no data available to verify this prediction.

\section{Discussion}

Cities have been around for many millennia, and have been an integral part of shaping our civilization. However, cities have also always been associated with crime.

Here we examine how the number of crimes in cities scales with city population, and present a descriptive mathematical framework that provides an explanation for it. It has been previously shown that crime scales super-linearly with city size (Bettencourt et al., 2007) (that is, larger cities have disproportionately more crime than smaller cities). It has been assumed that the super-linear scaling of crime is due to agglomerative processes. Such an explanation has also been given for scaling of GDP, patents and other outputs of super-creative professions (Bettencourt et al., 2007; Pan et al., 2013). Scaling of GDP and patents stems presumably from the fact that they are created by creative professionals who benefit from living in larger cities by the disproportionately higher number of innovative collaborations and connections larger cities provide (Youn et al., 2015). However, most criminals are not creative professionals, and hence they may not benefit from increasing returns to living in larger cities.

Our modelling and empirical data suggest that crime in cities scales super-linearly (Fig. 2) because of number of police scaling sublinearly with city size (Fig. 4) and crimes being generated linearly with city size (Fig. 3). Our models also make quantitative predictions of how crime should scale with city size and the estimates are in agreement with observed data. Our models suggest that the super-linear scaling of crime in cities is a result of the interplay between generation of crime and police numbers. This is supported by the fact that the number of requests for

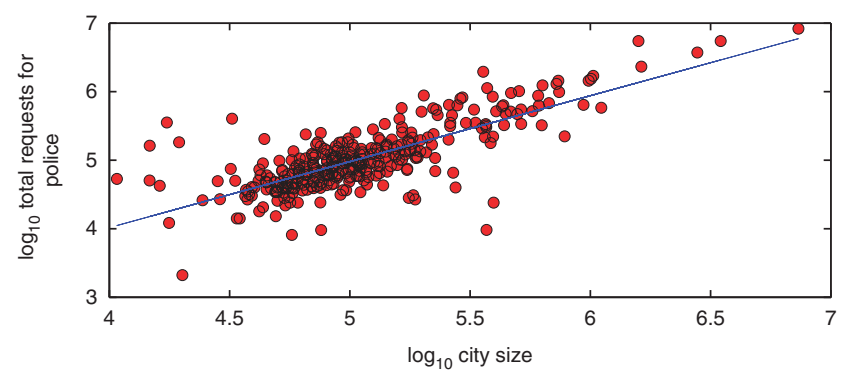

Figure 3 | Linear scaling of total requests for police in cities. Note: Plot showing total requests for police versus city size (population) from empirical data. OLS slope $=0.96, r^{2}=0.60, P$-value $=1 e-70$.

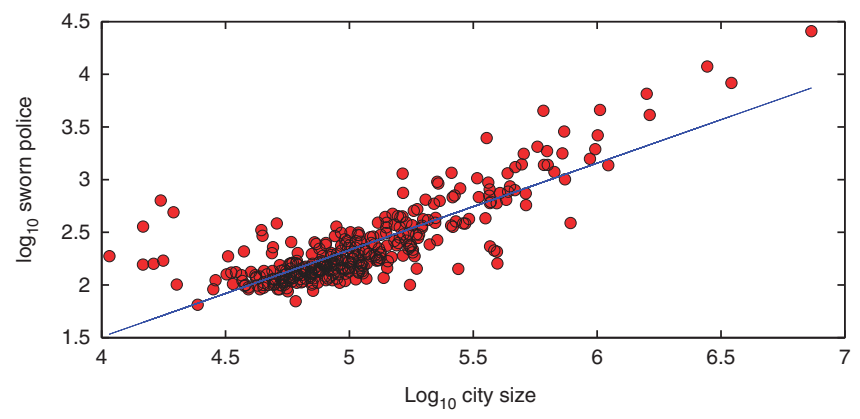

Figure 4 | Sublinear scaling of sworn police in cities. Note: Plot showing sworn police number versus city size (population) from empirical data. OLS slope $=0.82, r^{2}=0.69, P$-value $=5 e-88$.

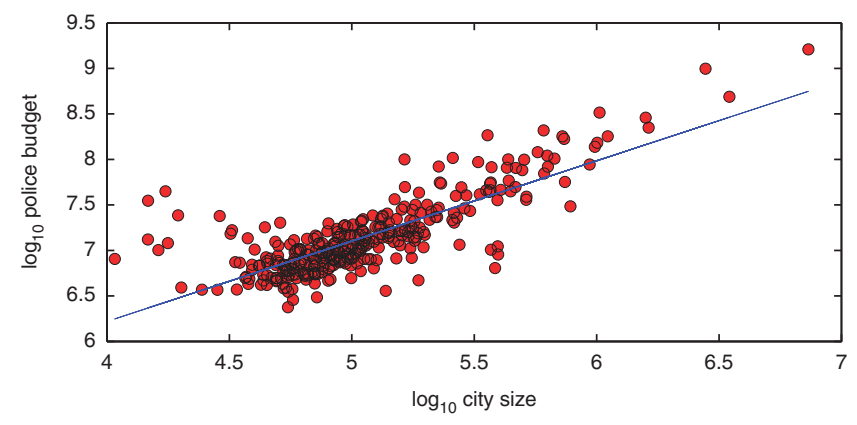

Figure 5 | Sublinear scaling of police budget in cities. Note: Plot showing police budget versus city size (population) from empirical data. OLS slope $=0.88, r^{2}=0.65, P$-value $=7 e-80$.

police support scales linearly with city size (Fig. 3, OLS slope $=0.96$, Table 1 ). These are requests for police during or immediately after a crime. Our data therefore suggests that crime is generated isometrically (linearly) with city size. However, the net crime (after considering police numbers and police response) scales super-linearly (Fig. 2, OLS slope $=1.26, r^{2}=0.65$ ).

Our mathematical model also correctly explains crime in a different context: in universities. Our data shows that net crime in universities scales sublinearly with the size of the university (Fig. 6 ). Since the number of police in universities scales sublinearly also (Fig. 7), we predict that the actual number of crimes generated is much lower. Our model (equation (4)) predicts that the number of police requests in universities must also have been sublinear, instead of the linear relationship observed in cities. Hence universities may not be attractive to criminals. We hypothesize that this is due to the fact that universities have intellectual capital and not financial capital and are less of an incentive for criminals than for example retail outlets (Davies et al., 2013). 


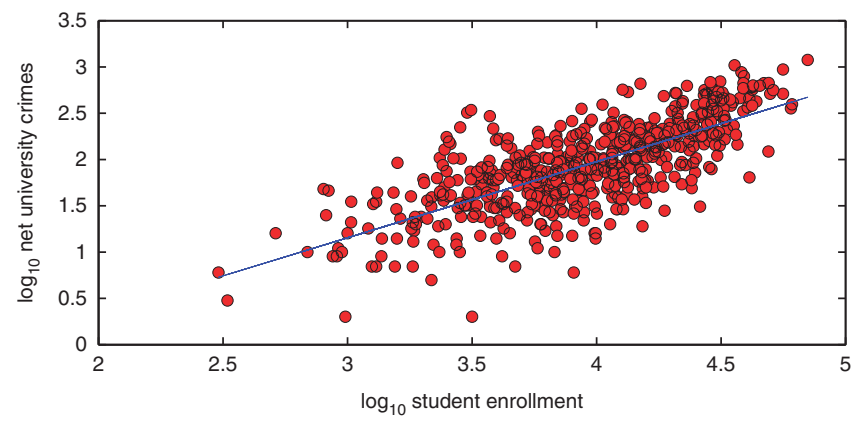

Figure 6 | Sublinear scaling of total crimes in universities. Note: Plot showing net crimes in universities versus university size (enrolment size) from empirical data. OLS slope $=0.82, r^{2}=0.53$. $(P$-value $=3 e-98)$.

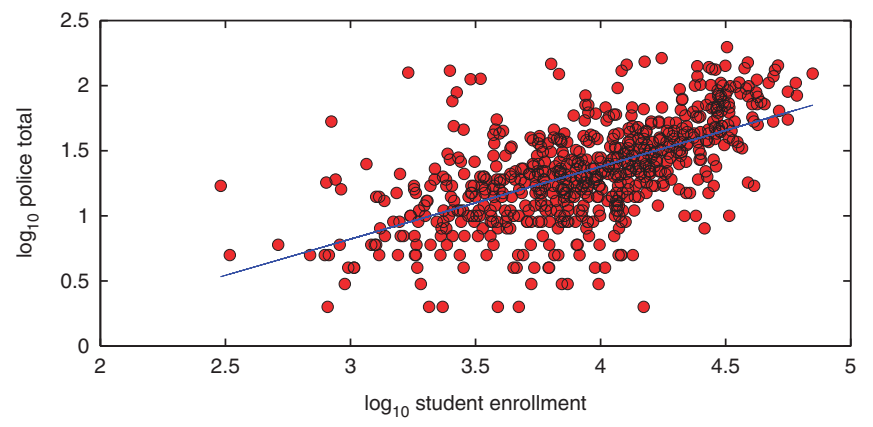

Figure 7 | Sublinear scaling of police in universities.

Note: Plot showing police in universities versus university size (enrolment size) from empirical data. OLS slope $=0.56, r^{2}=0.37$. $(P$-value $=$ 2e-66).

The present work points to deficiencies in police numbers: police numbers do not scale linearly with the population of a city (Fig. 4) and police budget scales sublinearly with city population (Fig. 5) (that is, police budget per capita is lower in larger cities). This raises the intriguing possibility that it may be possible to reduce crime in larger cities by allocating more police and crime fighting resources (by allocating more towards police budget). Our models suggest that if police numbers did scale linearly with city size, crimes could be reduced and net crimes would also scale linearly (and not superlinearly) with the size of the city (setting $x_{\text {crime }}=1$ and $y_{\text {police }}=1$ in equation (4)). We do not suggest, however that the mere presence of additional police or more efficient policing may serve to revert crime to a linear scaling regime, since attacks on criminal networks may in some cases strengthen them further (Duijn et al., 2014). Nevertheless, a judicious strategy that combines additional police with effective patrolling and crime prevention techniques may reduce crime in large cities.

Our models can also predict how net crime should scale if requests for police and police numbers scale differently than in the dataset shown here (by substituting the appropriate scaling relationships for $x_{\text {crime }}$ and $y_{\text {police }}$ in equation (4)). This could be useful in analysing other specialized forms of crime like cyber crime, organized crime, crime in armies and online vandalism (for example, misinformation in Wikipedia and so on).

The present work also suggests that criminals (for the crimes we investigate) and police are not able to take advantage of increasing returns (that is, criminals and police do not benefit from being in larger cities and may not be able to exploit the additional information and collaboration rich environments that they provide). This is in stark contrast to innovative and economic enterprise where people benefit disproportionately from living in larger cities that afford more avenues for collaboration (Bettencourt et al., 2007; Pan et al., 2013).

Our work raises important questions about crime itself: why do the number of crimes generated scale linearly with the population of a city and not say super-linearly? If the most important attractor for crime is money, and GDP itself scales super-linearly with size of the city (Bettencourt et al., 2007), crimes committed in the city should also scale super-linearly. It is possible that crimes are after all committed against individuals (of some financial value) and the total number of crimes scales only as the number of people available in the city (population or size of the city). Another likely cause is that this reflects fundamental constraints in how criminals operate: a single criminal has only so much cognitive and physical capability and can do a limited amount of damage in a specified amount of time. Unless criminals are able to exploit collaborations with other criminals, the intrinsic rate of generation of crime will always be isometric (linear) with the population of a city.

Cities are akin to biological organisms (Bettencourt et al., 2007); crime is analogous to a pathogenic infection and police response to it is similar to an immune response. Such an immune inspired theory or immunological theory of crime in cities has several advantages:

1. Previous work has shown advantages of looking at complex systems from the lens of another complex dynamical system: the immune system (Banerjee and Moses, 2009; Banerjee and Moses, 2010a,b; Banerjee et al., 2011; Moses and Banerjee, 2011; Banerjee, 2009, 2013). One of the advantages is that the observed scaling of a quantity of interest is the result of the complex nonlinear interplay between two different competing systems (pathogen and the immune system or criminals and police). This approach has also been used successfully to derive results for how the immune response against pathogens should scale with the size of the infected animal (Banerjee and Moses, 2009; Banerjee and Moses, 2010b; Banerjee et al., 2011; Banerjee, 2013).

2. Previous work on using the immune system as inspiration has also led to insights into design of engineered distributed systems of computers and mobile robots (Banerjee and Moses, 2010a, b; Moses and Banerjee, 2011; Banerjee, 2013). We hope that the present work would lay the foundation for an immunological theory of crime in cities. Such a theory may give insights into how crime, social unrest and civil disorder (analogous to pathogenic infection) develops in cities, a theory of attack against people or migrants considered foreign to the native population, civil wars where fighting develops within cities and fighting against intruders trying to invade cities. It could give insights into how some cities are able to defend themselves against intruders while still remaining flexible enough to incorporate new people with various skillsets that deeply enrich it. Such insights may help us design better and more efficient cities.

3. Finally, previous work using the immune system as an inspiration has shown the optimal way to design and place structures similar to lymph nodes (used by the biological immune system) that facilitate detection of adverse events and response against them (Banerjee and Moses, 2010a, b; Moses and Banerjee, 2011; Banerjee, 2013). In an immunological theory of cities, the analogue of lymph nodes would be police stations and patrolling police would be circulating lymphocytes (Banerjee et al., 2011). In future work we plan to extend the theory presented here by making theoretical predictions of optimal placement and size of police stations, and optimal police trafficking strategies. 
The biological immune system exhibits scale-invariance of detection and response (Banerjee and Moses, 2010a, b; Banerjee, 2013) (that is, the time taken by the immune system to detect a pathogen and neutralize it does not scale with the size of the organism). Our work suggests that if cities allocated more (linearly) resources towards police, then crime would scale linearly with the size of the city and hence the density of crime would be scale-invariant (from equation (4)). Organisms also allocate energy to the immune system proportional (linear) to their body size. This linear allocation of energy to the immune system is one reason (among other factors) that helps the immune system achieve scale-invariance (Banerjee and Moses, 2010a, b; Banerjee, 2013) and is likely the result of evolution, and the importance of the immune system to the survival of organisms.

Organisms may have evolved to minimize host immune response times and host tissue damage to pathogens (Banerjee and Moses, 2010a, b; Banerjee, 2013). This raises an intriguing question: why have cities not evolved to minimize crime? There are two potential explanations:

1. Cities may not have been subjected to the same evolutionary pressures, or for as long a time, as organisms. In addition, moving out of cities is costly, and that may keep cities stable.

2. Crime may not affect the attractiveness of a city as long as it is below some perceived threshold (that is, there are more important factors like economic opportunity that may overwhelm factors like crime when it comes to making a decision to move to a city).

In future work we plan to extend our approach to: (a) cyber crimes, where it is difficult to quantify size because of its decentralized nature, and (b) organized crime, where interaction and co-operation between criminals can lead to different dynamics. The present approach of modelling the competition and dynamics between two opposing forces can also be extended to model human conflicts and would be consistent with existing work demonstrating that conflict dynamics are best captured by co-evolution between aggressors (Johnson et al., 2011). Finally, our models ignore space and assume that a city composed of criminals, non-criminals and law enforcement is homogeneously well-mixed. However, space is very important in determining dynamics of crime (Short et al., 2010; Baudains et al., 2013c; Davies et al., 2013). Future work will couple spatially explicit models with dynamics between criminals, non-criminals and law enforcement.

In summary, our work suggests that super-linear crime scaling does not imply that crime generation is super-linear. The present work highlights the importance of incorporating non-linear dynamics while simulating complex systems like cities. Previous work has used a similar approach to study immune systems (Banerjee and Moses, 2010b; Banerjee, 2013).

Cities have been around for many millennia, have been an integral part of shaping the growth of civilizations and have contributed immensely to our success as a dominant and cognitive species. However, crime has been synonymous with cities since their inception. A deeper understanding of crime is imperative to the habitability of our planet, sustainable living in cities and our future success. We hope that this work will lay the foundation for a general and ultimately predictive theory for crime in cities.

\section{Note}

1 Supplementary Information accompanies this paper at http://www.palgrave-journals .com/palcomms

\section{References}

Banerjee S (2009) An Immune System Inspired Approach to Automated Program Verification. arXiv preprint arXiv:0905.2649.

Banerjee S (2013) Scaling in the immune system, PhD Thesis, University of New Mexico, USA.

Banerjee S, Levin D, Moses M, Koster F and Forrest S (2011) The value of inflammatory signals in adaptive immune responses. In: Lio, Pietro et al. (eds.) Artificial Immune Systems, 10th International Conference, ICARIS, Lecture Notes in Computer Science, Springer Verlag: Berlin, Germany, vol 6825, pp 1-14.

Banerjee S and Moses M (2009) A hybrid agent based and differential equation model of body size effects on pathogen replication and immune system response. In: P.S. Andrews et al. (eds) Artificial Immune Systems, 8th International Conference, ICARIS, 2009, Lecture Notes in Computer Science, Springer Verlag, Berlin: Germany, vol 5666, pp 14-18.

Banerjee S and Moses M (2010a) Modular RADAR: An immune system inspired search and response strategy for distributed systems. In: E. Hart et al. (eds) Artificial Immune Systems, 9th International Conference, ICARIS, 2010, Lecture Notes in Computer Science, Springer Verlag: Berlin, Germany, vol 6209, pp 116-129.

Banerjee $S$ and Moses M (2010b) Scale invariance of immune system response rates and times: Perspectives on immune system architecture and implications for artificial immune systems. Swarm Intelligence; 4 (4): 301-318.

Banerjee S, Van Hentenryck P and Cebrian M (2015) Replication Data for: Competitive dynamics between criminals and law enforcement explains the super-linear scaling of crime in cities, Dataverse. http://dx.doi.org/10.7910/ DVN/ELSYXO.

Batty M (2008) The size, scale, and shape of cities. Science; 319 (5864): 769-771.

Baudains P, Braithwaite A and Johnson S D (2013a) Spatial patterns in the 2011 London riots. Policing; 7 (1): 21-31.

Baudains P, Braithwaite A and Johnson S D (2013b) Target choice during extreme events: A discrete spatial choice model of the 2011 London riots. Criminology; 51 (2): 251-285.

Baudains P, Johnson S D and Braithwaite A M (2013c) Geographic patterns of diffusion in the 2011 London riots. Applied Geography; 45 (December), 211-219.

Bettencourt L, Lobo J, Helbing D, Kühnert C and West G (2007) Growth, innovation, scaling, and the pace of life in cities. Proceedings of the National Academy of Sciences of the United States of America; 104 (17): 7301-7306.

Bettencourt L M (2013) The origins of scaling in cities. Science; 340 (6139): 1438-1441.

Bettencourt L and West G (2010) A unified theory of urban living. Nature; 467 (7318): 912-913.

Cullen J B and Levitt S D (1999) Crime, urban flight, and the consequences for cities. Review of Economics and Statistics; 81 (2): 159-169.

Davies T P, Fry H M, Wilson A G and Bishop S R (2013) A mathematical model of the London riots and their policing. Scientific Reports; 3: 1303.

D'Orsogna M R and Perc M (2014) Statistical physics of crime: A review. Physics of Life Reviews; 12 (March): 1-21.

Duijn P A, Kashirin V and Sloot P M (2014) The relative ineffectiveness of criminal network disruption. Scientific Reports; 4: 4238.

Glaeser E (2011) Triumph of the City: How Our Greatest Invention Makes us Richer, Smarter, Greener, Healthier and Happier. Pan Macmillan: London.

Glaeser E and Sacerdote B (1996) Why is there more crime in cities? Journal of Political Economy; 107 (6): 225-258.

Gould E D, Weinberg B A and Mustard D B (2002) Crime rates and local labor market opportunities in the United States: 1979-1997. Review of Economics and Statistics; 84 (1): 45-61.

Helbing D et al (2014) Saving human lives: What complexity science and information systems can contribute. Journal of Statistical Physics; 158 (3): 735-781.

Johnson N et al (2011) Pattern in escalations in insurgent and terrorist activity. Science; 333 (6038): 81-84.

Keizer K, Lindenberg S and Steg L (2008) The spreading of disorder. Science; 322 (5908): 1681-1685.

Lichman M (2013) 'UCI machine learning repository'. University of California, Irvine, School of Information and Computer Sciences. http://archive.ics.uci.edu/ml

MATLAB and Statistics Toolbox Release (2012) The MathWorks, Inc., Natick, Massachusetts, United States.

McMillon D, Simon C P and Morenoff J (2014) Modeling the underlying dynamics of the spread of crime. PloS One; 9 (4): e88923.

Moses M and Banerjee S (2011) Biologically Inspired Design Principles for Scalable, Robust, Adaptive, Decentralized Search and Automated Response (RADAR). Proceedings of the 2011 IEEE Conference on Artificial Life, pp. 30-37. 
Pan W, Ghoshal G, Krumme C, Cebrian M and Pentland A (2013) Urban characteristics attributable to density-driven tie formation. Nat. Comm; 4: 1961.

Perc M, Donnay K and Helbing D (2013) Understanding recurrent crime as system-immanent collective behavior. PloS One; 8 (10): e76063.

Redmond M and Baveja A (2002) A data-driven software tool for enabling cooperative information sharing among police departments. European Journal of Operational Research; 141 (3): 660-678.

Salesses P, Schechtner K and Hidalgo C A (2013) The collaborative image of the city: Mapping the inequality of urban perception. PloS One; 8 (7): e68400.

Schläpfer M et al (2014) The scaling of human interactions with city size. Journal of The Royal Society Interface; 11 (98): 20130789.

Short M B, Brantingham P J, Bertozzi A L and Tita G E (2010) Dissipation and displacement of hotspots in reaction-diffusion models of crime. Proceedings of the National Academy of Sciences of the United States of America; 107 (9): 3961-3965.

Tumminello M, Edling C, Liljeros F, Mantegna R N and Sarnecki J (2013) The phenomenology of specialization of criminal suspects. PloS One; 8 (5): e64703.

United States Department of Commerce, Bureau of the Census, Census of Population and Housing 1990 United States: Summary Tape File 1a \& 3a (Computer Files). (1990) URL: http://archive.ics.uci.edu/ml/datasets/Commu nities+and+Crime, accessed 1 September 2014.

United States Department of Justice, Bureau of Justice Statistics, Law Enforcement Management And Administrative Statistics (Computer File). (1992) URL: http://archive.ics.uci.edu/ml/datasets/Communities+and+Crime, accessed 1 September 2014.

United States Department of Justice, Federal Bureau of Investigation, Crime in the United States (Computer File). (1995) URL: http://archive.ics.uci.edu/ml/ datasets/Communities+and+Crime, accessed 1 September 2014.

Warton D I, Duursma R A, Falster D S and Taskinen S (2012) SMATR 3 - An R package for estimation and inference about allometric lines. Methods in Ecology and Evolution; 3 (2): 257-259.

Warton D I, Wright I J, Falster D S and Westoby M (2006) Bivariate line-fitting methods for allometry. Biological Reviews of the Cambridge Philosophical Society Cambridge Philosophical Society; 81 (2): 259-91.

Youn H, Bettencourt L, Strumsky D and Lobo J (2015) Invention as a combinatorial process: Evidence from US patents. Journal of The Royal Society Interface; 12 (106): 20150272.

\section{Data Availability}

The datasets analysed during the current study are available in the Palgrave Communications' Dataverse repository (Banerjee et al., 2015): http://dx.doi.org/10.7910/DVN/ ELSYXO.

These datasets were derived from the following public domain resources: http://archive. ics.uci.edu/ml/datasets/Communities+and+Crime;

https://www.fbi.gov/about-us/cjis/ucr/crime-in-the-u.s/2011/crime-in-the-u.s.-2011/ tables/table-79-1/view;

https://www.fbi.gov/about-us/cjis/ucr/crime-in-the-u.s/2011/crime-in-the-u.s.-2011/ tables/table-9/view

\section{Acknowledgements}

SB acknowledges financial support from the National Information and Communications Technology Australia (NICTA) in sponsoring a research visit during which this project was initiated. PVH and MC are funded by the Australian Government as represented by the Department of Broadband, Communications and the Digital Economy and the Australian Research Council through the ICT Centre of Excellence program. The authors also wish to thank Dr. Michael Gastner for suggesting the use of semi-major axis regression for testing superlinear scaling.

\section{Additional Information}

Supplementary Information: accompanies this paper at http://www.palgrave-journals .com/palcomms

Competing interests: The authors declare no competing financial interests.

Reprints and permission information is available at http://www.palgrave-journals.com/ pal/authors/rights_and_permissions.html

How to cite this article: Banerjee S, Van Hentenryck P and Cebrian M (2015) Competitive dynamics between criminals and law enforcement explains the super-linear scaling of crime in cities. Palgrave Communications. 1:15022 doi: 10.1057/ palcomms.2015.22

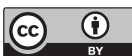

This work is licensed under a Creative Commons Attribution 3.0 International License. The images or other third party material in this article are included in the article's Creative Commons license, unless indicated otherwise in the credit line; if the material is not included under the Creative Commons license, users will need to obtain permission from the license holder to reproduce the material To view a copy of this license, visit http://creativecommons.org/licenses/by/3.0/ 